\title{
Hydraulic External Pre-Isolator System for LIGO
}

\author{
S Wen ${ }^{1}, \mathbf{R}$ Mittleman ${ }^{4}$, K Mason $^{4}$, J Giaime $^{1}, \mathbf{R}$ Abbott $^{1}$, \\ J Kern ${ }^{1}$, B O'Reilly ${ }^{1}$, R Bork ${ }^{3}$, M Hammond ${ }^{1}$, C \\ Hardham $^{2}$, B Lantz ${ }^{2}$, W Hua ${ }^{2}$, D Coyne ${ }^{3}$, G Traylor ${ }^{1}$, H \\ Overmier $^{1}$, T Evans ${ }^{1}$, J Hanson ${ }^{1}$, O Spjeld ${ }^{1}$, M Macinnis ${ }^{4}$, \\ K Mailand ${ }^{3}$, D Sellers ${ }^{1}$, K Carter $^{1}$ and P Sarin ${ }^{4}$ \\ ${ }^{1}$ LIGO Livingston Observatory, Box 940, Livingston, LA 70754, USA \\ ${ }^{2}$ Ginzton Laboratory, Stanford University, Stanford, CA 94305, USA \\ ${ }^{3}$ LIGO Laboratory, MS 18-34, California Institute of Technology, Pasadena, CA \\ 91125, USA \\ ${ }^{4}$ LIGO Laboratory, Bldg. NW17-161, Massachusetts Institute of Technology, \\ Cambridge, MA 02139, USA \\ E-mail: swen@ligo-la.caltech.edu
}

\begin{abstract}
The Hydraulic External Pre-Isolator (HEPI) is the first 6 degrees of freedom active seismic isolation system implemented at the Laser Interferometer Gravitational Wave Observatory (LIGO). Implementation was first completed at the LIGO Livingston Observatory (LLO) prior to LIGO's 5th science run, successfully cutting down the disturbance seen by LLO's suspended optics in the two most prominent seismic disturbance bands, the microseism $(0.1-0.3 \mathrm{~Hz})$ and the anthropogenic $(1-3 \mathrm{~Hz})$ bands, by a factor of a few to tens. The improvement in seismic isolation contributed directly to LLO's much improved duty cycle of $66.7 \%$ and LIGO's triple coincident duty cycle of $53 \%$. We report the design, control scheme, and isolation performance of HEPI at LLO in this paper. Aided with this success, funding for incorporating HEPI into the LIGO Hanford Observatory was approved and installation is currently underway.

PACS numbers: 04.80.Nn, 47.85.Kn, 43.40.Tm
\end{abstract}

\section{Introduction}

The Laser Interferometer Gravitational Wave Observatory (LIGO) hosts the world's most sensitive gravitational wave (GW) detectors capable of detecting strain of $h_{r m s}=10^{-22} / \sqrt{H z}$ at $100-200 \mathrm{~Hz}$. Its initial design consists of a power recycled Michelson interferometer with $4 \mathrm{~km}$ long Fabry-Pérot arm cavities, and with the output port operating slightly off a total dark fringe. Input light is a $1064 \mathrm{~nm} 10 \mathrm{~W}$ frequency and intensity stabilized solid-state Nd:YAG laser. The light is filtered by a $12 \mathrm{~m}$ triangular spatial mode cleaner downstreams, allowing only the fundamental mode to enter the interferometer. Main optics are isolated from ground disturbances passively by cascading stages of damped mass-spring stacks 1 and pendulum systems. Each stage provides an $\sim 1 / f^{2}$ attenuation well above their resonant frequencies. Five groups of co-located shadow sensor and magnet-coil actuator are attached to the face and side of each suspended optic, to provide translational and angular sensing and actuation. Through precision digital feedback control of the optics, angular alignment of the optics and lengths of various interferometer cavities are maintained. The 
optics and their supporting isolation systems are placed inside interconnected Basic Symmetric Chambers (BSCs) and Horizontal Access Modules (HAMs) under high vacuum. The BSCs host the beam splitter and the input and end test masses, while the HAMs host the input mode cleaner, recycling mirror, and the output optics.

At LIGO's designed sensitivity the noise curve is dominated by the attenuated seismic noise, mechanical resonances, and various technical noises below $40 \mathrm{~Hz}$, thermal noise between $40-100 \mathrm{~Hz}$, and photon shot noise above $100 \mathrm{~Hz}$. From 40 to $7000 \mathrm{~Hz}$, the suspended optics approximate free test masses in inertial space, and is the GW detection band. Passing GWs perturb the local space-time metric at the interferometer. The two differentially strained arm cavities cause their light to exist with a phase difference and the power of the interference at the output port is proportional to the differential length changes. Through a differential arm control loop, dark fringe is maintained, and it is the error signal and carefully calibrated parameters of the loop from which the GW signal is extracted. An important indicator of LIGO's sensitivity is the detection range for a $1.4-1.4 \mathrm{M}_{\odot}$ binary neutron star inspiral system, which for the $4 \mathrm{~km}$ detectors, exceeds $15 \mathrm{Mpc}$ with signal to noise ratio of 8 when averaged over all orientations and sky positions.

Such sensitivity was not achieved initially when LIGO was first built, but only after series of engineering and science runs, identifying and reducing noise sources along the way, and making incremental improvements for the past decade [2, 3, 4, 5]. Among the major upgrades were the elevated input laser power, evolved alignment and sensing control scheme, thermal compensation system [6], DC readout [7, and last but not least, the Hydraulic External Pre-Isolator System (HEPI).

At LIGO, we denote the state of the interferometer in 'science mode' when it reaches designed sensitivity, and the process of acquiring science mode 'lock acquisition'. The percentage of time LIGO is in science mode is the 'duty cycle'. Since any GW detection must be confirmed by at least two independent interferometers in order to reject local false event, scientific output of LIGO is maximized only when the duty cycle is high. This was proven difficult for the LIGO Livingston Observatory (LLO) prior to the 4th science run due to its noisy surrounding environment 8]. LLO was built near to the Gulf of Mexico, logging industries, and the town of Livingston. Oceanic and human activities periodically and aperiodically provide large ground disturbances at LLO in the microseism $(0.1-0.3 \mathrm{~Hz})$ and anthropogenic $(1-3 \mathrm{~Hz})$ bands, respectively. While these bands do not lie in LIGO's detection band, excitation puts a tremendous force, total range, and noise requirement on the optics actuators. The excitation was further worsened by the isolation stacks, while designed to provide attenuation in the detection band, have their lowest resonant modes overlapped with the anthropogenic band. When the ground motion was above the $\sim 1 \mu \mathrm{m} / \mathrm{s}$ threshold, science mode could not be maintained. It was not uncommon to see an entire daytime dedicated to lock acquisition for many days in a row. At night, multiple cargo trains passed through the town of Livingston, each knocking the interferometer out of science mode and preventing re-locking for about an hour. Duty cycle for the 3rd science run (S3) was merely $21.8 \%$. Finally, non-linear behavior of the interferometer's response to low-frequency seismic noise was found to degrade the sensitivity in the detection band [9. A separate stage of seismic isolation system in addition to the existing passive system was necessary for uninterrupted operation of the observatory, and HEPI was developed in such mist of struggle for duty cycle.

Prior to HEPI, the only effort to equip LLO with an external active system was 
the retrofit of the piezoelectric fine actuator system (FAS) [10. Performance of the system was relatively band and phase-accuracy limited, and was installed only for the chambers that host the input and end test masses. However, the success of the FAS inferred that the potential benefit a 6 degrees of freedom (DOFs) active isolation system brings can be tremendous. Several other mechanical, electromagnetic, piezoelectric, and magnetostrictive actuator designs were considered alongside the HEPI actuator, but were not selected due to frictional loss, hysteresis behavior, excess noise, and limited range. Final selection was made to the HEPI actuator for being able to avoid the aforementioned disadvantages and meet in particular the following requirements: bandwidth of more than $20 \mathrm{~Hz}$, noise of $10^{-10} \mathrm{~m} / \sqrt{\mathrm{Hz}}$ from 1 to $10 \mathrm{~Hz}$, and payload capacity of $2000 \mathrm{~kg}$. Early prototyping and control design of the HEPI system were developed at the Ginzton Laboratory at Stanford University and the LIGO Advanced System Test Interferometer at Massachusetts Institute of Technology [11, 12, 13, 14, 15. We report the the final implementation at LLO prior to the 5th science run (S5).

\section{Installation}

The HEPI system was installed around LLO's all five BSCs and HAM1-4. There are four HEPI actuator-sensor assemblies per chamber, located between the top of the four support piers and the ends of the two support beams. Each actuator-sensor assembly consists of hydraulic actuators, Kaman DIT-5200 position sensors, Mark $\mathrm{L}-4 \mathrm{C} 1 \mathrm{~Hz}$ geophones mounted in the vertical and horizontal directions, and also two off-load springs. The actuators and offload springs connect to a common actuation block, which serves as an interface between HEPI and the support beam. Position sensors and geophones were installed in-line with their corresponding actuator, the former located at the top of the actuator and the later inside the actuation block. Figure 1 and figure 2 illustrate the installation.

The off-load springs support the static weight of the suspended payload and were also used to coarsely position the payload. The position sensors sense the relative displacement between ground and the payload, and the geophones sense the payload's inertial velocity. Each HEPI actuator-sensor assembly can provide an actuation range of $\pm 1 \mathrm{~mm}$ in both vertical and horizontal directions. On the BSCs, the horizontal actuator-sensor axis is 45 degrees relative to the beamline direction $(\mathrm{X})$. On the HAMs, the angle is 30 degrees. Appropriate combinations of the actuators forces and sensor signals provide actuation and sensing of the payload in all six and also the horizontal and vertical over-constrained (HO and VO) DOFs.

The hydraulic actuators are in essence servo valve controlled pistons. A distinct feature of the actuators is their bellow design which eliminates frictional and hysteresis losses associated with traditional hydraulic pistons. Pressurized viscous fluid is supplied from central pump stations, filtered by two stages of micron level filters, and distributed to individual actuator through a network of clean piping. Differential pressure between supply and return lines is maintained by a simple PID control. Supply lines are fitted with accumulators to damp the noise from the pumps. The core of of the actuator control is the Parker DYP2SC servo valve, which is a hydraulic Wheatstone bridge and has its internal flow nozzles modified to maintain laminar flow in the system. The valve controls the differential pressure (and hence the delivered net force) between the two axially soft bellows and has a near-linear response to electrical 


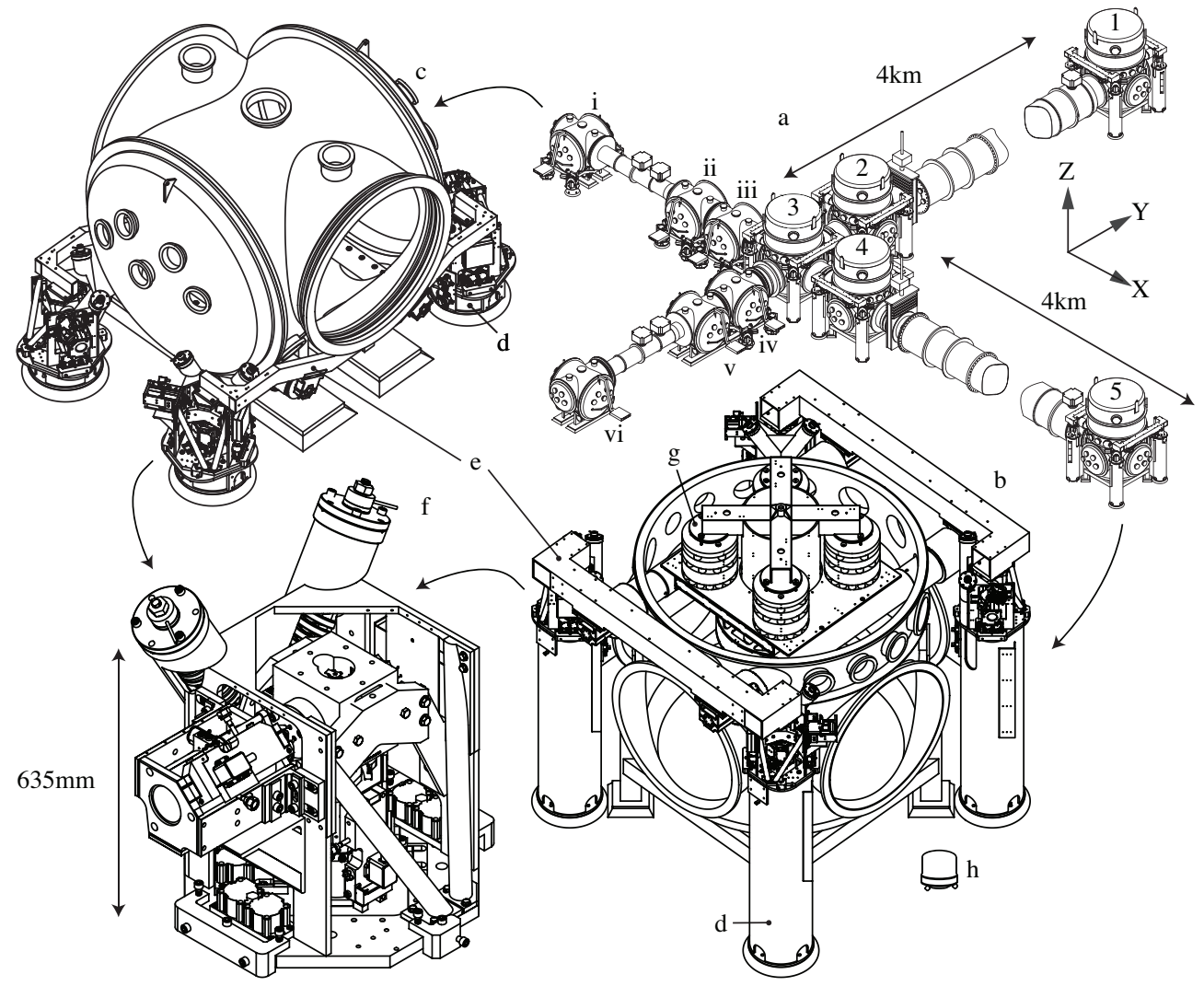

Figure 1. HEPI Installation at LLO. (a) 4km vacuum envelope, (b) BSC (BSC15 marked by 1-5), (c) HAM (HAM1-6 marked by i-vi), (d) support pier, (e) support beam, (f) HEPI actuator-sensor assembly, (g) isolation stacks, and (h) STS-2 seismometer.

control current. An actuation plate situated between the bellows transmits the force through an axially stiff (but otherwise soft) tripod to the payload. The flexibility of the bellows and tripod allow a pair of vertical and horizontal actuators to operate individually or simultaneously without being over-constrained. To damp the bellows' expansion resonances, internal hydraulic damping mechanisms were added between the bellows. Design parameters of the actuator is described in [14 in detail.

Finally, inseparable from the HEPI system are the three Streckeisen broadband $(0.01-50 \mathrm{~Hz})$, low-noise, 3-axis STS-2 seismometers, which are placed on the ground of LLO's corner and two end stations to sense the local seismic disturbance.

\section{Control design}

The control design of HEPI consists of position sensor and geophone feedback loops, and seismometer feed-forward paths. Position sensor loops must work with feedforward paths to achieve isolation, while for the geophone feedback loops it can be achieved working alone. Payload position sensed by the DIT-5200 position sensors is filtered and used to command the actuators, which in turn move the payload, forming 


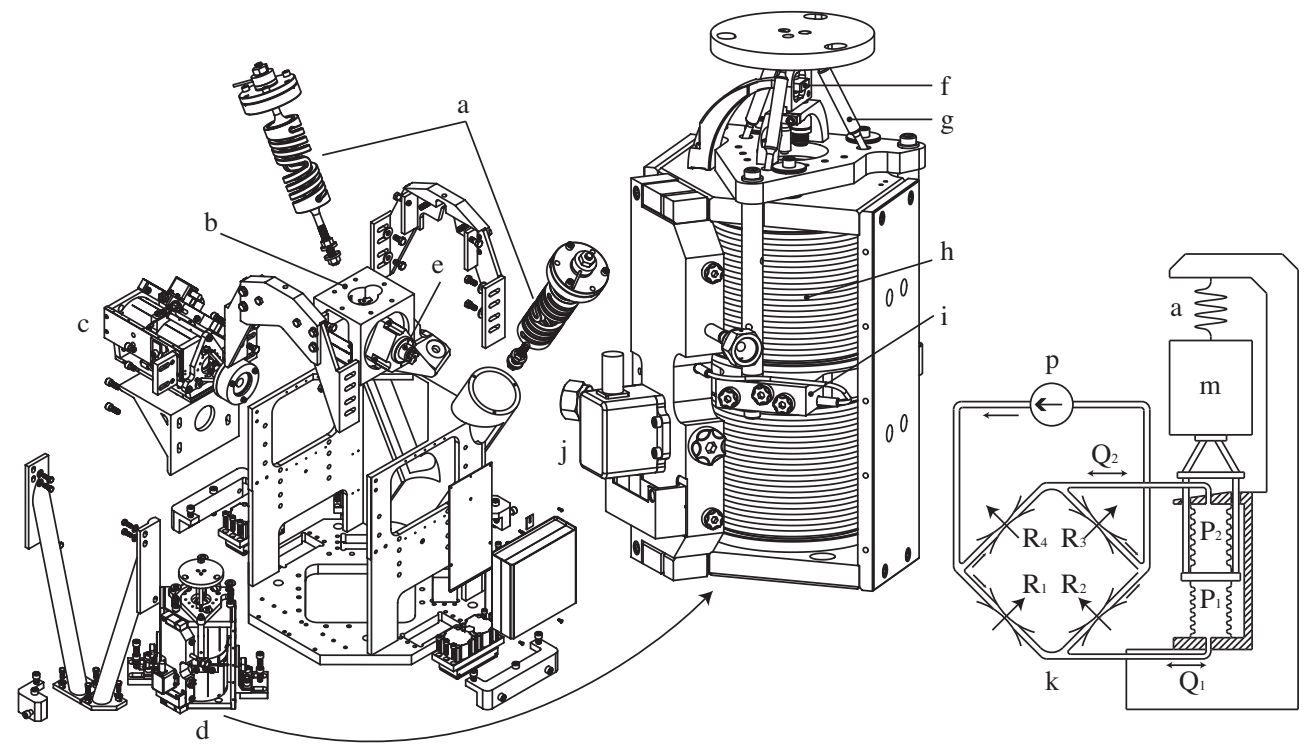

Figure 2. Exploded view of the actuator-sensor assembly and schematic of actuator hydraulics. (a) Offload springs, (b) interface to payload, (c) horizontal actuator, (d) vertical actuator, (e) L-4C geophone, (f) position sensor, (g) tripod, (h) bellow, (i) actuation plate, (j) DYP2SC control valve, (k) hydraulic bridge, (m) payload, and (p) pump.

feedback loops which control the payload position with respect to the ground. Ground motion sensed by the STS-2 seismometers is feed-forwarded (subtraction) into the error points of the position feedback loops, commanding the payload to move by the same amount but in opposite direction as the ground motion, suppressing the ground to payload transfer function. Since the STS-2 only senses motion in the three translational directions, feed-forward is possible only in those directions as well. Payload motion sensed by the $\mathrm{L}-4 \mathrm{C}$ geophones is processed in the same fashion as the position, forming feedback loops which suppress the payload motion. Signal rate is $2048 \mathrm{~Hz}$ throughout the system.

For a given DOF, when both types of feedback loops are used, we customarily call the loops 'blended', and the frequency at which the responses of the two sensors have equal magnitude is called the 'blending frequency'. The synthetic sensor formed by two sensors and their filters is called the 'super sensor'. Figure 3 illustrates the control diagram for a DOF with blended feedback loops and a feed-forward path. Not every DOF has their loops blended. When not blended, the geophone loop is not used, and the position sensor filter is not needed since the compensator serves the same function.

Table 1 summarizes the implemented control strategy of the BSCs for all DOFs during S5. As a first iteration, X, Y, and Z had blended loops and feed-forward paths to make the most of HEPI's isolation capability. RX and RY were controlled by position sensor loops only, to suppress horizontal actuation to tilt coupling. RZ was blended since this DOF directly couples to the optics yaw alignment. HO and VO were also controlled by position sensor loops only, to suppress any noise coupling from those DOFs. The overall structure of the control was sigle-input-single-output. 


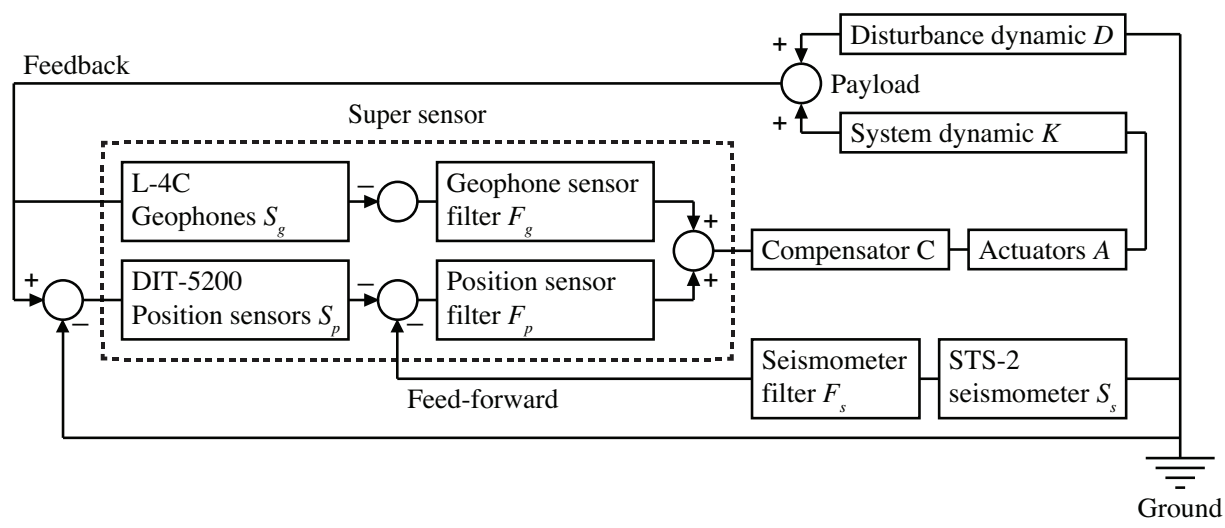

Figure 3. Control diagram of HEPI system.

Table 1. Control strategy of HEPI system for different DOFs. A prefix R indicates rotation about an axis. Fields with '-' are not implementable.

\begin{tabular}{cccccc}
\hline & \multicolumn{3}{c}{ BSC } & & \multicolumn{2}{c}{ HAM } \\
\cline { 2 - 3 } \cline { 5 - 5 } & Blended & Feed-forward & Blended & Feed-forward \\
\hline $\mathrm{X}$ & $\checkmark$ & $\checkmark$ & $x$ & $\checkmark$ \\
$\mathrm{Y}$ & $\checkmark$ & $\checkmark$ & & $x$ & $\checkmark$ \\
$\mathrm{Z}$ & $\checkmark$ & $\checkmark$ & $x$ & $\checkmark$ \\
$\mathrm{RX}$ & $x$ & - & $x$ & - \\
$\mathrm{RY}$ & $x$ & - & $x$ & - \\
$\mathrm{RZ}$ & $\checkmark$ & - & $x$ & - \\
$\mathrm{HO}$ & $x$ & - & $x$ & - \\
VO & $x$ & - & $x$ & - \\
\hline
\end{tabular}

Sensed motion of the payload and ground in a certain DOF was used to control the payload in that DOF only. The control strategy of the HAMs was modified from that of the BSCs' for reasons explained at the end of this section.

The transfer function from the ground to the payload is,

$$
T=\frac{D+G_{p}\left(1-\left(G_{s} / S_{p}\right)\right)}{1+G_{g}+G_{p}},
$$

where $G_{p}=S_{p} F_{p} C A K$ and $G_{s}=S_{s} F_{s} C A K$, are the position sensor and geophone feedback loop gains, respectively, and $G_{s}=S_{s} F_{s}$ is the feed-forward gain. Minimizing $T$ is achieved by either elevating $G_{g}$, or properly designing an $F_{s}$ that zeros the numerator. System stability is determined by the denominator $\left(1+G_{g}+G_{p}\right)$, with values further away from the complex zero being more stable.

Judging from (11), one may ask why not have either feedback or feed-forward work alone to achieve isolation. In practice, due to the different sensitivity range of the inertial sensors, cross-coupling behavior of the system, and imperfect design of $G_{s}$, it is impossible to have either perform well for the entire frequency range of interest $(0.1-10 \mathrm{~Hz})$, hence the need for blended loops to take advantage of the best of both controls. Maximizing the isolation performance while minimizing the noise entering the system determines the blending frequency. We found setting the blending frequency at $0.5 \mathrm{~Hz}$ yields the best result. Below the blending frequency, feed-forward out-performs feedback. Above it, the situation is reversed. 

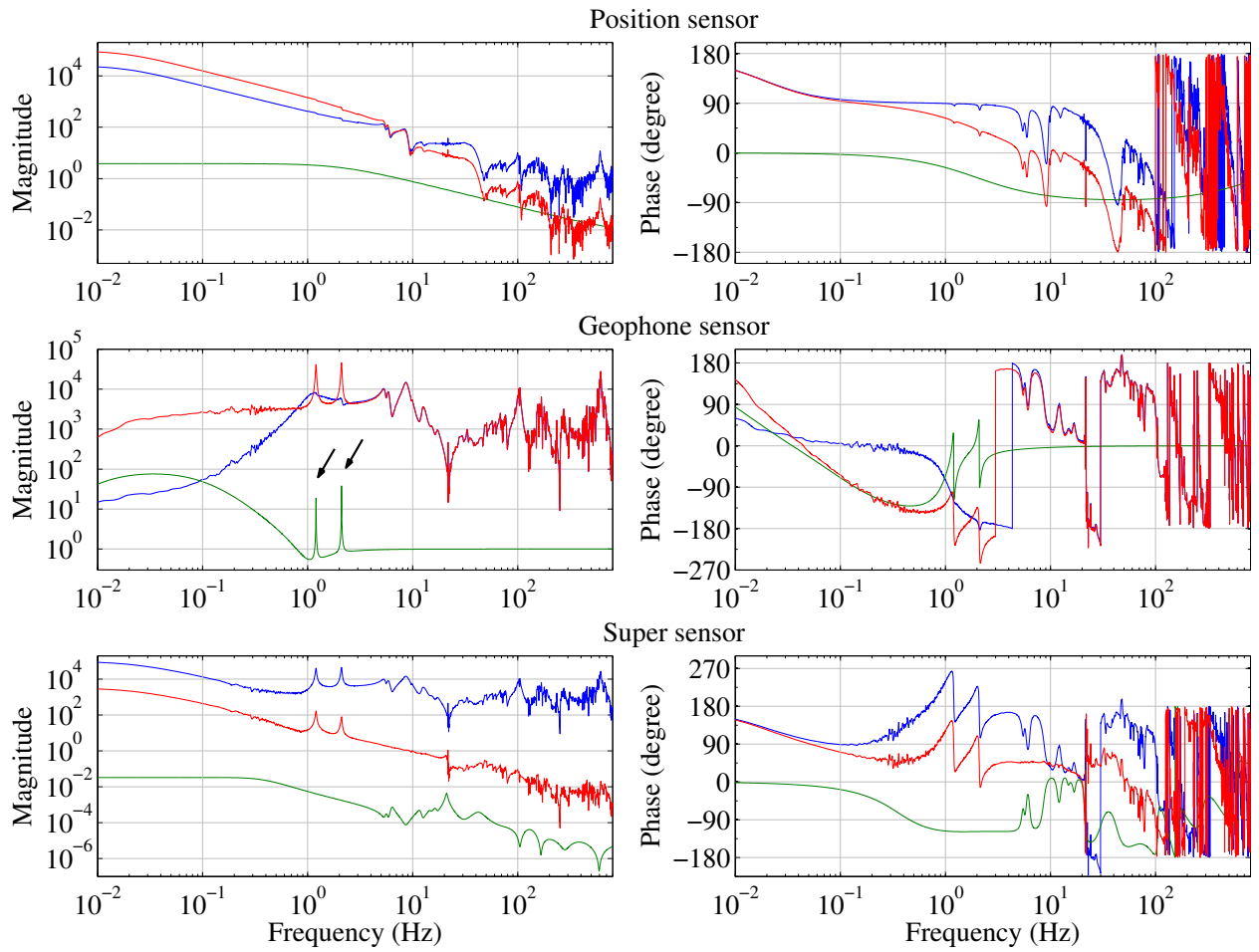

Figure 4. Control design of HEPI system. Blue traces: actuator to sensor transfer functions; Green: sensor filters; Red: filtered transfer functions. The extra 'boost' in gain is marked by arrows.

Control design for HEPI was done entirely in the frequency domain. We started with system identification, by driving the actuators with sinusoidal signal at frequencies ranging from 0.01 to $800 \mathrm{~Hz}$, and recording the sensor responses. Below the blending frequency, the geophone response was attenuated. Above it, the position sensor response was attenuated. Figure 4 illustrates such design. At the lowest resonant frequencies of the isolation stacks, the geophone loop gain was given an additional 'boost' at the cost of altered phase, and had the effect of further lowering the ground to suspended optics transfer function. To appreciate the effect, it is worth pointing out that these resonances, typically at 1.2 and $2.1 \mathrm{~Hz}$, lies within the anthropogenic band, and can be excited easily daily, potentially costing downtime of the interferometer.

The super sensor filter, or compensator, design was done semi-automatically. Below $20 \mathrm{~Hz}$, lower order modes of the isolation stacks were easily compensated. The response data was chunked into several frequency ranges, each fitted with minimum number of poles and zeros following the least sum of squared error method. The results from each frequency range were stitched together and inverted. Then, alternating real zeros and poles were added to augment the gain and maintain the phase. From 20 to $80 \mathrm{~Hz}$, there is a broadband feature which includes the actuator bellow expansion mode, actuator tripod compression mode, support beam flexing mode, support pier horizontal vibration mode, and many other fine mechanical resonances. They were 

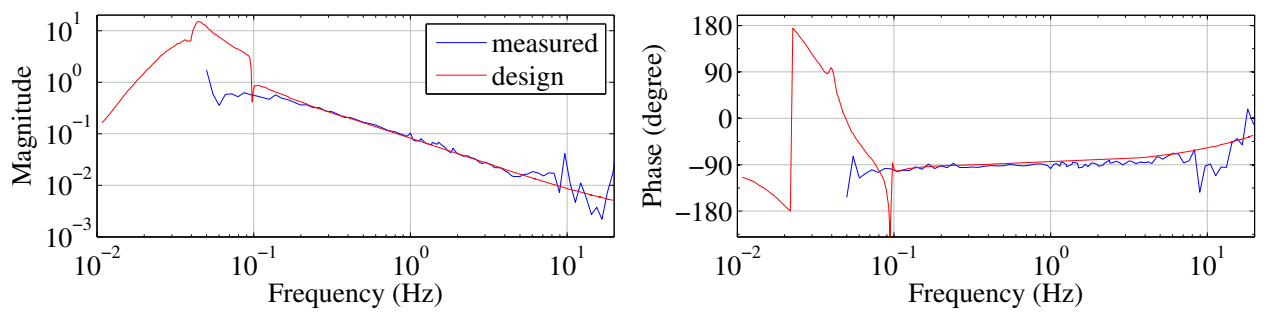

Figure 5. Feed-forward filter design. The $\sim 1 / f$ curve above $0.1 \mathrm{~Hz}$ originates from converting the seismometer output signal, in velocity, to position.

seldom resolved well enough to have them compensated perfectly, and this was the primary limitation of setting the control loop unity gain frequency (ugf) above $20 \mathrm{~Hz}$. Above $80 \mathrm{~Hz}$, resonances are too numerous to be characterized individually. Alternating complex zeros and poles were added to attenuate the response and prevent system oscillation or instability, with zeros aligning with the response poles and vice versa. Overall, the rule of thumb we applied was allowing 45 degrees of phase margin at and below the ugf at $10 \mathrm{~Hz}$, and a factor of 3 of gain margin above it. This put the slope of the loop gain below ugf at roughly $1 / f^{1.5}$.

A perfect design of the seismometer filter $F_{s}$ has a value,

$$
F_{s}=\frac{S_{p}}{S_{s}}\left(1+\frac{D}{G_{p}}\right)
$$

This quantity was measured directly with a two-step process. First the system was brought up to a feedback controlled state, but without the feed-forward path engaged. We measured the ground to payload transfer function which ideally would yield,

$$
t_{1}=\frac{D+G_{p}}{S_{s}\left(1+G_{g}+G_{p}\right)} .
$$

Then, we drove at the error point of the position sensor loop with a magnitude much greater than the ground noise level. The transfer function from the drive to the payload is,

$$
t_{1}=\frac{G_{p} / S_{p}}{1+G_{g}+G_{p}} .
$$

The ratio between the two, $t_{1} / t_{2}$, is exactly the $F_{s}$ sought after, and the remaining task was fitting the $F_{s}$. This was the design method above $0.1 \mathrm{~Hz}$.

An intrinsic property of the seismometer is that at low frequencies, the horizontal sensing component is also sensitive to ground tilt with a $1 / f^{2}$ dependency. To avoid the tilt-contaminated horizontal ground signal from being feed-forwarded into the system and generates unwanted payload motion, the seismometer filter $F_{s}$ incorporates a $0.1 \mathrm{~Hz}$ high pass polyphase complementary filter [15, 16. Such a filter has a $f^{2}$ roll-off below $0.04 \mathrm{~Hz}$, gain of one and phase of zero above $0.1 \mathrm{~Hz}$, with a constant gain of 4 between 0.04 and $0.1 \mathrm{~Hz}$ as a trade-off. The effect is less in the vertical direction and such high pass filter is not needed. Figure 5 illustrates the overall filter design for the horizontal directions.

The initial control strategy for the HAMs was identical to that of the BSCs'. However, it was soon discovered that due to the relatively smaller cross-sectional size, shorter span, and geometry of the HAM external support beams, actuating the HAMs 
in the $\mathrm{X}$ direction twists the ends of the support beams and tilts the entire platform in the RY direction, at a magnitude of $0.1 \mathrm{rad} / \mathrm{m}$. As a result, the geophones also sense the incorrect induced motion. Blending was abandoned for the HAMs. Instead, the geophone signal paths were cut-off entirely from the feedback portion of the control, only the position sensor signal paths were used. Isolation for the HAMs was achieved by feed-forward paths only. In addition, to compensate the actuation-induced tilt, an extra feed-forward path was added, by taking the output of the feed-forward signal in the $\mathrm{X}$ direction, and feeding it to the error point of the position sensor loop in the $\mathrm{RY}$ direction.

In the corner station, in order to minimize the disturbance to the mode cleaner cavity length caused by the differential motion of HAM1 and HAM2, identical feedforward signals in the beamline direction were fed into both HAMs. The same tactic was implemented for the power recycling cavity hosted by HAM3 and BSC3. However, these were done as a first order approximation without further considering the subtle difference between the chambers' responses to feed-forward signal, and assuming the ground motion is unchanged over the length of the cavity.

\section{Isolation performance and stability}

We evaluated the isolation performance of HEPI at two stages, the HEPI stage and the final suspended optics. Performances did not vary much among different BSCs and among different HAMs, due to their structural and control resemblance. Typical performances of the BSCs and HAMs from 0.01 to $50 \mathrm{~Hz}$ are shown in figure 6 and figure 7, while the band-limited rms reduction is shown in table 2. Features within the control design clearly manifested themselves in the performance figures. For instance, the 'boost' in the geophone filter drastically reduced the horizontal transmission at the lowest isolation stack mode resonant frequencies, while the trade-off made in the feed-forward filter design between 0.04 and $1 \mathrm{~Hz}$ increased it. Notice that for the HAMs, in order to get the basic isolation improvement at the suspended optics, the noise at the HEPI stage was enhanced due to the intentional tilt correction.

Above $0.1 \mathrm{~Hz}$, the benefit of improved isolation on cavity length noise was dependent on the type of chamber in which the cavity mirrors were hosted. A cavity with both mirrors in the BSCs received the most improvement, while that in the HAMs improved only above and worsen below $0.5 \mathrm{~Hz}$. A cavity with mirrors in both the BSC and HAM had improvement level in-between the previous two types (figure 8 and table 33). Below $0.01 \mathrm{~Hz}$, the first two types were also affected by the trade-off mentioned previously. This diverse behavior clearly indicated that neither individually designing feed-forward filters for the chambers nor forcing their feedforward signals to be identical is an optimal solution to reducing cavity length noise.

Over the course of $\sim 6$ years which encompassed three science runs, HEPI proved to be stable in a temperature-controlled environment of 20 degree Celcius. We did not observe any apparent frequency response changes. Chemical analysis of the HEPI hydraulic fluid revealed an unexpected high concentration of boron. However, there was no clear indication of degraded physical properties of the fluid nor compromise in isolation performance. There were few incidences where the small passages of the actuator control valve were clogged up by microscopic debris in the fluid. The valves were quickly replaced with spares and only led to few hours of observatory downtime. 

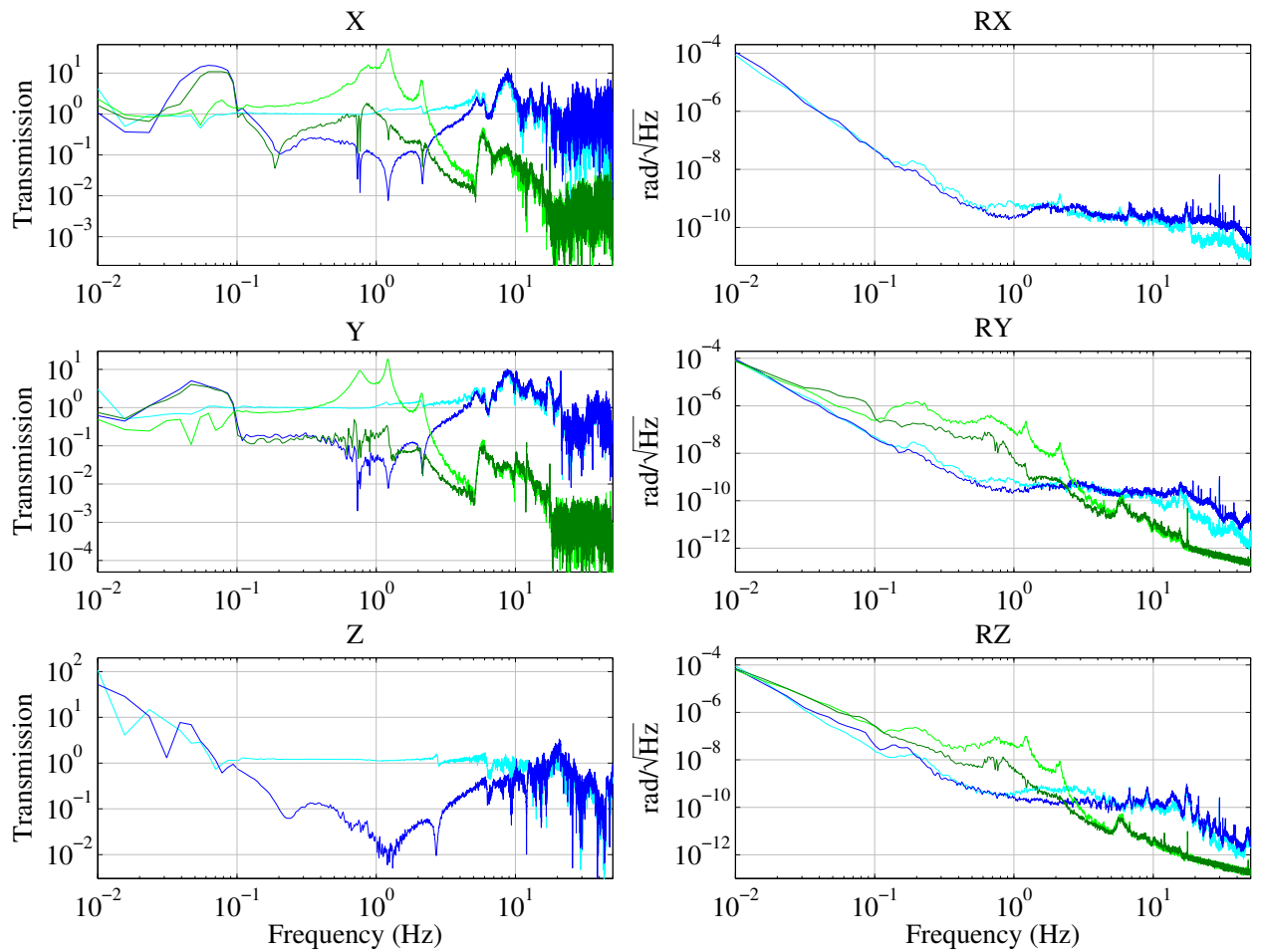

Figure 6. Typical BSC isolation performance. Light blue traces: HEPI stage, HEPI turned off. Dark blue traces: HEPI stage, HEPI turned on. Light green traces: suspended optics, HEPI turned off. Dark green traces: suspended optics, HEPI turned on.

Table 2. Average BSC and HAM isolation performance in the $0.1-0.3$ and $1-3 \mathrm{~Hz}$ bands. For X, Y, and Z DOFs, it is expressed as factor of reduction in the rms transmission from the ground to the HEPI level and suspended optics. For RX, RY, and RZ DOFs, it is the factor of reduction in the rms amplitude noise. Values greater than one indicate improvement. Fields with '-' are not measurable.

\begin{tabular}{|c|c|c|c|c|c|c|c|c|}
\hline & \multicolumn{4}{|c|}{$\mathrm{BSC}$} & \multicolumn{4}{|c|}{ HAM } \\
\hline & \multicolumn{2}{|c|}{ HEPI } & \multicolumn{2}{|c|}{ Optics } & \multicolumn{2}{|c|}{ HEPI } & \multicolumn{2}{|c|}{ Optics } \\
\hline & $0.1-0.3 \mathrm{~Hz}$ & $1-3 \mathrm{~Hz}$ & $0.1-0.3 \mathrm{~Hz}$ & $1-3 \mathrm{~Hz}$ & $0.1-0.3 \mathrm{~Hz}$ & $1-3 \mathrm{~Hz}$ & $0.1-0.3 \mathrm{~Hz}$ & $1-3 \mathrm{~Hz}$ \\
\hline $\mathrm{X}$ & 2.94 & 8.51 & 4.20 & 21.80 & 0.35 & 5.62 & 8.11 & 10.94 \\
\hline $\mathrm{Y}$ & 6.08 & 15.99 & 5.68 & 26.76 & 0.78 & 1.28 & 6.01 & 6.87 \\
\hline $\mathrm{Z}$ & 7.89 & 30.99 & - & - & 17.91 & 1.42 & - & - \\
\hline RX & 1.07 & 2.69 & 4.13 & 23.21 & 0.74 & 0.73 & - & - \\
\hline RY & 2.08 & 2.02 & 5.04 & 26.41 & 0.12 & 0.36 & 3.63 & 4.18 \\
\hline $\mathrm{RZ}$ & 1.05 & 4.69 & 2.01 & 26.08 & 0.40 & 2.08 & 1.36 & 4.70 \\
\hline
\end{tabular}

\section{Improvement in duty cycle}

Success of the HEPI system at LLO marked a major improvement in duty cycle, from $21.8 \%$ of S3 to $66.7 \%$ of S5. Figure 9 shows the percentage of time the LIGO Hanford Observatory (LHO) $4 \mathrm{~km}$ and LLO detectors were in science mode under different seismic noise conditions in the $0.1-0.1 \mathrm{~Hz}$ and $1-3 \mathrm{~Hz}$ bands-the two major 

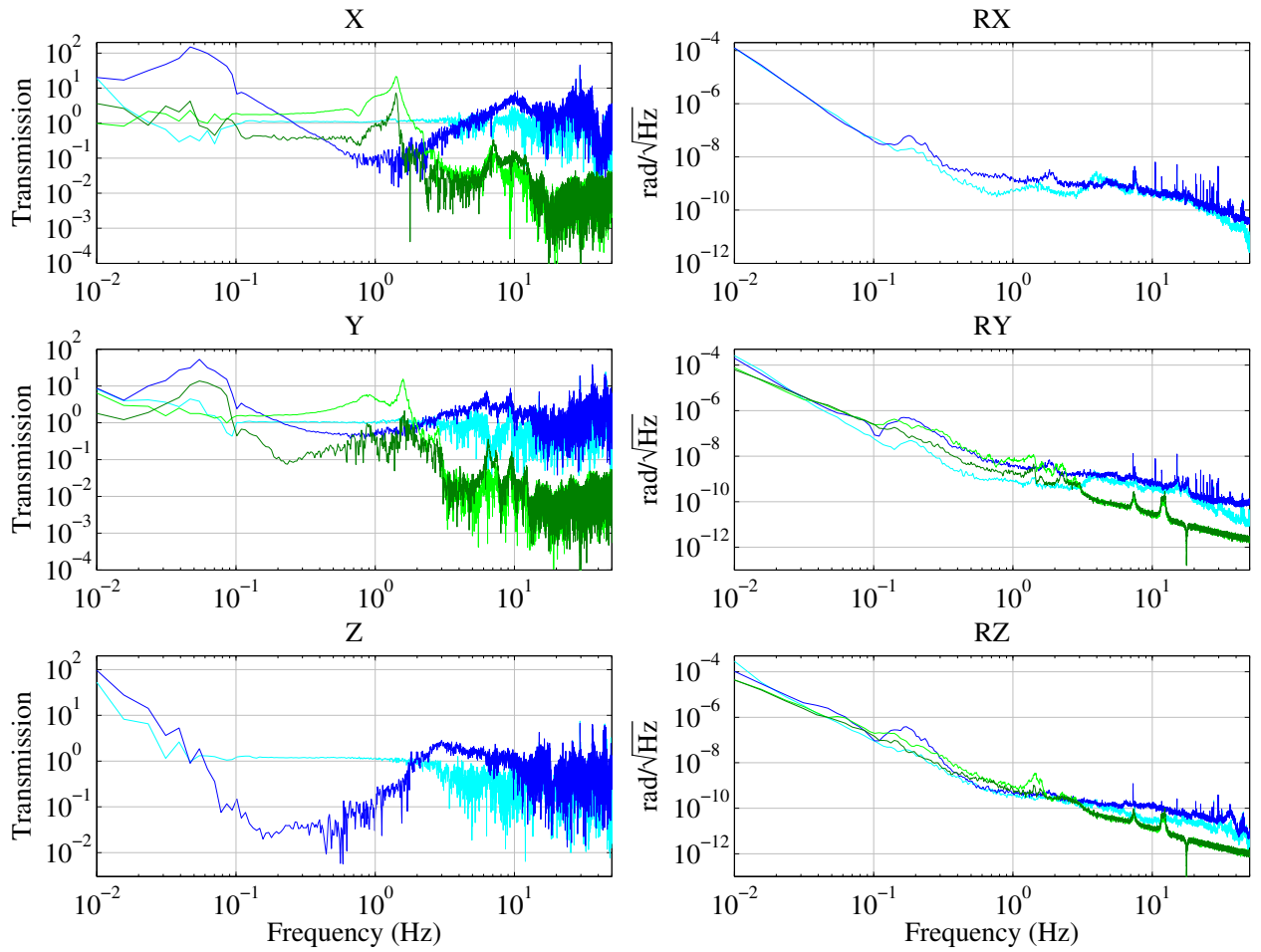

Figure 7. Typical HAM isolation performance. Light blue traces: HEPI stage, HEPI turned off. Dark blue traces: HEPI stage, HEPI turned on. Light green traces: suspended optics, HEPI turned off. Dark green traces: suspended optics, HEPI turned on.
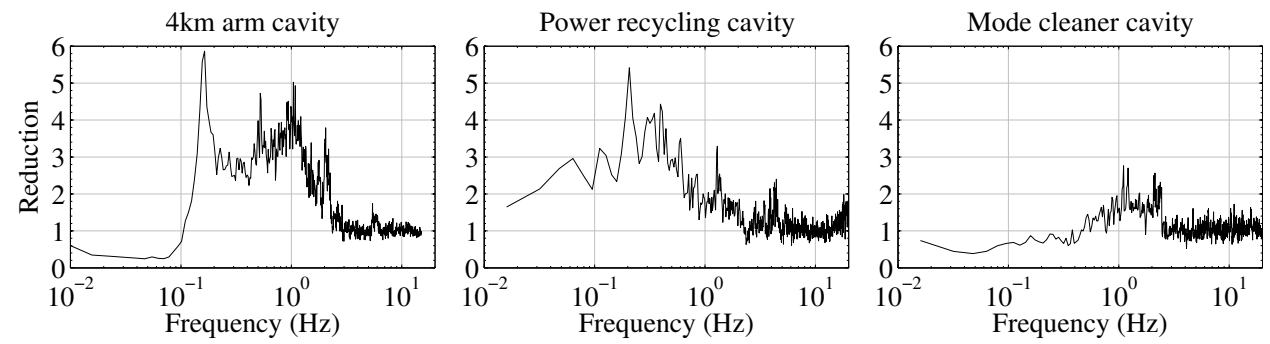

Figure 8. Reduction in cavity length noise. From left to right panel, the mirrors of the indicated cavities are hosted by two BSCs, one BSC and one HAM, and two HAMs, respectively. Values greater than one indicate improvement.

Table 3. Factor of reduction in rms cavity length noise in the $0.1-0.3$ and $1-3 \mathrm{~Hz}$ bands.

\begin{tabular}{lcc}
\hline Cavity & $0.1-0.3 \mathrm{~Hz}$ & $1-3 \mathrm{~Hz}$ \\
\hline 4km arm & 2.82 & 2.65 \\
Power recycling & 3.48 & 1.23 \\
Mode cleaner & 0.83 & 1.30 \\
\hline
\end{tabular}




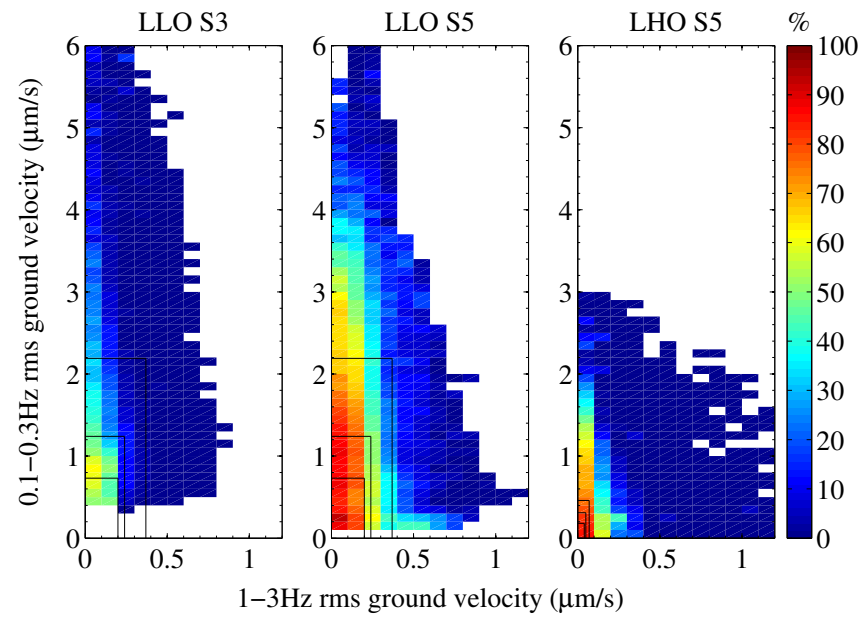

Figure 9. Comparison of science mode statistics at both sites, evaluated at the frequency bands indicated by the axes. Color in each velocity bin indicates rate of reaching science mode. Boxes mark the 50th, 75 th, and 90 th velocity percentiles. Bins with white color indicate no such data ever recorded.

Table 4. Minimum rate (\%) of obtaining science mode when rms ground velocity in both the $0.1-0.3$ and $1-3 \mathrm{~Hz}$ bands is under given velocity percentile.

\begin{tabular}{cccccc}
\hline Velocity & \multicolumn{2}{c}{ LLO } & & \multicolumn{2}{c}{ LHO } \\
\cline { 2 - 3 } \cline { 5 - 5 } percentile & S3 & S5 & & S5 & Projected \\
\hline 50 th & 44 & 74 & 83 & 88 \\
75 th & 0 & 50 & 79 & 85 \\
90 th & 0 & 15 & 76 & 83 \\
\hline
\end{tabular}

bands HEPI was designed to target. Result suggests that in S5, the velocity at which LLO could maintain science mode for at least $50 \%$ of the time was roughly three times of that in S3 in either band, provided the velocity in the other band was held at the lowest level. The number was roughly twice when compared with LHO in S5. At LLO, when the ground condition fell within the boundary formed by the 50th velocity percentile of each band, the minimum rate of reaching science mode improved form $44 \%$ in S3 to $74 \%$ in S5. Rates for other velocity percentiles for LLO and LHO are summarized in table 4 .

An iconic improvement to LLO was its locking ability during train passes (figure 10). For such events, a separate high-noise locking state was devised for the interferometer. The noise floor of such state was orders of magnitude higher than that of science mode, and the data gathered was unsuitable for GW detection. However, maintaining in such state cut down the time required to re-acquire science mode lock, as oppose to starting from an uncontrolled state. Trains that caused less than $\sim 0.6 \mu \mathrm{m} / \mathrm{s}$ (accounting for $\sim 50 \%$ of all trains) in the $1-3 \mathrm{~Hz}$ band did not break lock. Previously with the FAS system, differential arm length correction could not exceed $90 \mu \mathrm{m}$, beyond which the interferometer broke lock as it lost track of a particular wavefront in the arms. HEPI extended the range to $0.7 \mathrm{~mm}$, reducing the downtime associated with such constraint.

It is interesting to know what LHO's duty cycle would be with HEPI installed. 

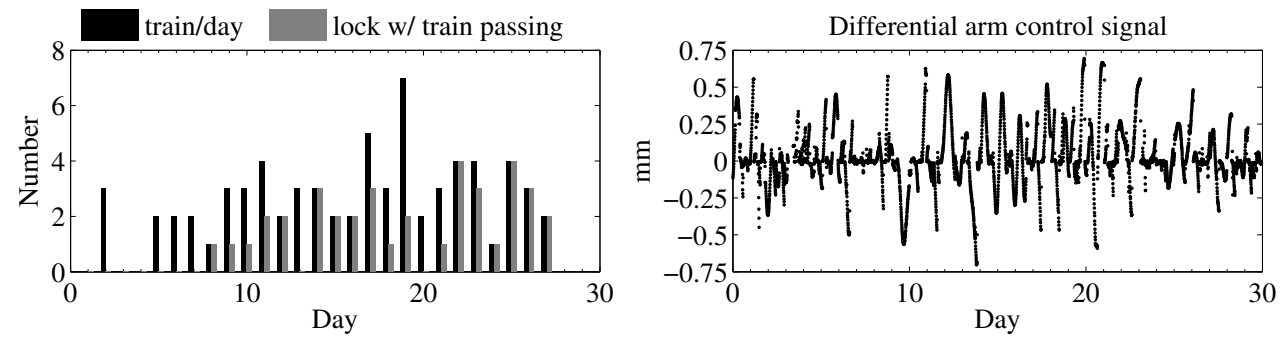

Figure 10. A sample of interferometer lock during train passes and differential arm control signal over a 30-day period.

This was estimated by applying the LLO's S5 science mode statistics towards LHO's ground velocity distribution profile. Our estimation delivered $86.2 \%$ duty cycle, or about a full month of additional science data per year. The projected improvement is also shown in table 4. We expect actual figure to be even higher for the coming 7th science run, as Advanced LIGO will also incorporate the in-vacuum two-stage active isolation system [16, 17, which covers the $0.1-10 \mathrm{~Hz}$ band.

\section{Conclusion and future work}

We demonstrated that employing simple feedback and feed-forward controls, a DOFdependent isolation factor of a few to tens can be easily achieved by the BSC HEPI system. We also identified that the poorer performance of the HAM mostly originated from the softness of its external support beam structure. Improvement in cavity length noise was chamber-type dependent. Overall effect was the factor of $\sim 3$ improvement in duty cycle.

The control techniques learned at LLO can be applied towards HEPI's Advance LIGO [18] installation. Improvement will largely depend on identifying the remaining noise sources, their paths, and fine tuning the controls. More sophisticated control schemes such as adaptive and Wiener filtering have been experimented to some extent at LASTI, but the improvement in isolation is yet to be fully characterized before their final implementation at the observatories. Further improvement in cavity length noise using the existing HEPI system has been demonstrated in [19]. Extending the isolation to below $0.1 \mathrm{~Hz}$ requires proper removal of the tilt noise intrinsic to the seismometer response. Once successful, it has the potential of improving the duty cycle during high wind periods which tend to cause the ground motion to peak at below $0.1 \mathrm{~Hz}$. Investigation of the ground tilt noise at LIGO as well as the requirement and selection of possible tilt instruments is specified in 20. The effort opens up the possibility of incorporating blended loops and feed-forward in the rotational DOFs, reducing the rotational-to-translational coupling of the seismic isolation to suspension systems.

Future hardware work includes installing HEPI on HAM5 and 6, which will host the output mode cleaner [7 for Advanced LIGO. Plans for modifying and testing the HAM support beams were carried out at LASTI and will be incorporated into following HEPI upgrades. Finally, the promising performance of HEPI prompted identical installation at LHO. Installation of the pump station and distribution piping is complete as well as manufacturing most of the HEPI actuator-sensor assembly 
mechanical parts. Parts underwent more stringent testing requirements than those of initial installation at LLO. Repeatability and accuracy of manufacturing the actuator bellows remain a challenge, as they depend highly on the manufacturing process and labor skills. We fine tuned both to ensure the bellows meet the requirement.

\section{Acknowledgments}

The authors acknowledge financial support from National Science Foundation through grants PHY-0071316, 0107417, 0140297, 0304924, and 9801158, as well as Louisiana Board of Regents contract LEQSF(2000-03)-RD-A-06.

\section{References}

[1] Giaime J, Saha P, Shoemaker D and Sievers L 1996 Review of Scientific Instruments 67 208-214

[2] Richard L Savage J 1998 Proc. SPIE Methods for Ultrasensitive Detection 3270 2-13

[3] Raab F J 2004 Proc. SPIE 5500, Gravitational Wave and Particle Astrophysics Detectors 5500 $11-24$

[4] Sigg D and the LIGO Science Collaboration 2006 Classical and Quantum Gravity 23 S51

[5] Abbott B P et al 2009 Reports on Progress in Physics 72076901

[6] Willems P 2009 in Adaptive Optics: Methods, Analysis and Applications (Optical Society of America) p. AOThA5

[7] Fricke T T et al 2012 Classical and Quantum Gravity 29065005

[8] Daw E J, Giaime J A, Lormand D, Lubinski M and Zweizig J 2004 Class. Quantum Grav. 21 2255

[9] Ottaway D J, Fritschel P and Waldman S J 2012 Opt. Express 20 8329-36

[10] Giaime J A, Daw E J, Weitz M, Adhikari R, Fritschel P, Abbott R, Bork R and Heefner J 2003 Rev. Sci. Instrum. 74 218-24

[11] Abbott R et al 2002 Class. Quantum Grav. 191591

[12] Robertson N A et al 2004 Proc. SPIE Gravitational Wave and Particle Astrophysics Detectors $\mathbf{5 5 0 0} 81-91$

[13] Abbott R et al 2004 Class. Quantum Grav. 21 S915

[14] Hardham C et al 2004 Proc. ASPE Spring 2004 Topical Meeting on Control of Precision Systems 32 127-32

[15] Hua W, DeBra D B, Hardham C T and Lantz B T 2004 Proc. ASPE Spring 2004 Topical Meeting on Control of Precision Systems 32 109-14

[16] Hua W et al 2004 Proc. SPIE Gravitational Wave and Particle Astrophysics Detectors 5500 194-205

[17] Matichard F et al 2010 Proc. ASPE Spring 2010 Topical Meeting on Control of Precision Systems 75-80

[18] Harry G M and the LIGO Scientific Collaboration 2010 Classical and Quantum Gravity 27 084006

[19] DeRosa R, Driggers J C, Atkinson D, Miao H, Frolov V, Landry M, Giaime J A and Adhikari R X 2012 Class. Quantum Grav. 29215008

[20] Lantz B, Schofield R, OReilly B, Clark D E and DeBra D 2009 Bull. Seismol. Soc. Am. 99 980-9 